\title{
Research on the inter-partition PID control algorithm of variable mass pitching systems
}

\author{
Zi-Fan FANG ${ }^{1, b}$, Zeng-Wu MA ${ }^{1, a^{*}}$, Xu-Fu GE ${ }^{1}$, Wei-Hua YANG ${ }^{1}$, Zi-Fan \\ FANG $^{2}$ \\ ${ }^{1}$ Mechanic and Material College, China Three Gorges University, Yichang, China \\ ${ }^{2}$ Hubei Key Laboratory of Hydroelectric Machinery Design \& Maintenance, China Three Gorges \\ University, Yichang, China \\ a1020709129@qq.com, bfzf@ctgu.edu.cn \\ ${ }^{*}$ Corresponding author
}

Keywords: variable mass pitching system;Three-loop control system;inter-partition PID control algorithm; joint simulation

Abstract.The variable mass pitching systems along with load quality and its pitch angle displacement, and the same as its position of the center of the pitching and driving torque changes etc.To design the adaptive control strategy, we put forward the inter-partition PID control algorithm for the driven motor of the pitching mechanism. To position servo precision and follow-up time as the goal, according to the pitch rate feedback, three interval PID control algorithm is designed. Based on ADAMS and Simulink simulation technology, we established the virtual prototype model of variable quality pitching system. Through simulation and physical prototype test, the classical PID and the inter-partition PID control algorithm are compared and analyzed by the position control accuracy and rapidity. The results show that, within the scope of the regulation response time, the error precision is less than $0.5^{\circ}$, the inter-partition PID control algorithm can effectively improve the control accuracy of the variable mass pitching system.

\section{Introduction}

With industrial development, the pitching system is widely used in different fields,such as the weapon equipment, radar, engineering machinery, lifting transportation machinery, forest fire, robots and other mechanical equipment.Most of the equipment are affected by space load (such as force, torque, etc.) and unexpected shocks, and even the equipment itself is a variable quality system $^{[1]}$.In the study of simulation, due to the load of variable mass system exists serious nonlinear,under different motion state, the load model parameters change is bigger, and the moment of inertia exists great uncertainty,makes mathematical model is difficult to accurately reflect actual motion control in the process of motion features and attributes of controlled object. Therefore, it is particularly important to establish the simulation model and the position servo control strategy of variable mass pitching system.

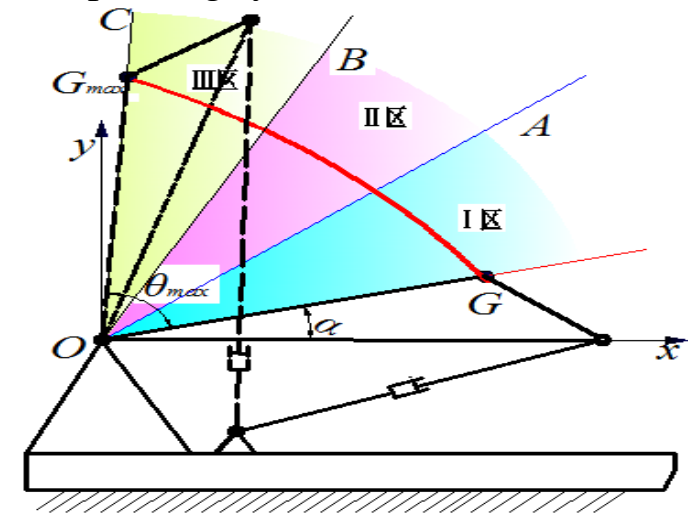

Fig. 1 Variable mass pitch system mechanism diagram 
As shown in Figure 1 is a variable mass pitching system schematic, the pitch system is mainly composed of a base, a pitch drive oil cylinder and a pitch body. the $\mathrm{xOy}$ coordinate system is established, $G$ denotes the gravity of pitch body, $\theta$ denotes the pitch angle of the system at any time, and the change of pitch angle displacement at any moment is equal to the change of angular displacement of center of mass, a denotes the structure-angle of pitch body's centroid, $\angle \mathrm{GOD}$ denotes the maximum pitch angle $\theta \max$ of the pitch change. Under varying load conditions, through a large number of simulation experiments show that it is very difficult to achieve the desired results in the same set of control parameters of the classical PID controller. In order to find suitable control parameters, the artificial pitch angle is divided into three intervals which denote I,II,III , OA, OB, OC denote respectively upper boundary of three districts, the arc GGmax represents movement trajectory of pitch body's centroid. As shown in Table 1 in different range, pitch angle drive torque is changed, so the position servo control strategy is put forward higher requirements [2-5].

Through a lot of simulation experiments have found that when the pitch angle is in different range, some parameters can be compensated and corrected, which can lead to more close to the expected effect. In this paper, we propose the inter-partition PID control algorithm location tracking method for variable mass pitching systems, which is based on a class of thermal engineering objects with parameter uncertainties.

Table.1. Change process of driving torque parameters in different range of pitch

\begin{tabular}{|c|c|c|c|}
\hline Interval & I & II & III \\
\hline Driving torque & $\begin{array}{l}\text { The initial value is larger, } \\
\text { gradually decrease }\end{array}$ & $\begin{array}{l}\text { Gradually in } \\
\text { crease }\end{array}$ & $\begin{array}{l}\text { Gradually } \\
\text { decrease }\end{array}$ \\
\hline
\end{tabular}

\section{Three loop control strategy of inter partition PID}

Control strategy. A typical three closed-loop PID control system is composed of the position loop, speed loop and current loop, as shown in Figure 2, according to the requirements of the system, Selecting DC motor as the drive motor, adopts three closed loop PID control system to improve the position of the variable mass pitching system response accuracy. The difference of position setting and position detection signal through the position regulator output, as the given speed; the difference of peed set point and the speed detection signal through the speed regulator output, as a given current; current loop as the inner loop, which can quickly track reference current. The current loop and speed loop are given respectively from the speed feedback and position feedback, position loop are given from the motor output speed, and current after the pulse width modulation (PWM) get control voltage, to control the motor speed, motor through a speed reducer drives the load inertia, in order to achieve the purpose of position control.

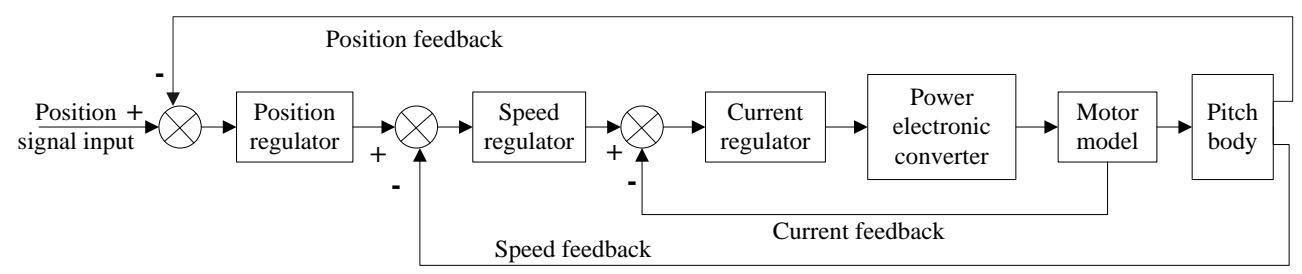

Fig.2. Diagram of pitch drive electromechanical system

Three Loop Control System Design. Design of current loop regulator. The main effect of the current loop is to limit the current, so the following is particularly important; the current loop is generally corrected into a typical I type system. Current loop PI controller transfer function is:

$$
W_{A C R}(s)=K_{p i}+\frac{K_{i i}}{s}=\frac{K_{p i}\left(\tau_{i} s+1\right)}{\tau_{i} s}
$$


Here, Kpi is the current loop regulator ratio coefficient; $\tau \mathrm{i}$ is the current loop regulator time constant; Kii is the integral time coefficient of the current loop regulator.

The open loop transfer functions of the current:

$$
W_{o p i}=\frac{I(s)}{I^{*}(s) / \gamma}=\frac{K_{p i}\left(\tau_{i} s+1\right)}{\tau_{i} s} \frac{\beta K_{s} / R_{a}}{\left(T_{l} s+1\right)\left(T_{\sum i^{i}} s+1\right)}
$$

Here, $K_{s}$ is the amplification factor of PWM; $\beta$ is the current feedback coefficient; $R_{a}$ is the armature winding.

Design of speed loop regulator. Generally, the velocity loop is corrected to typical II type system .The transfer function of the speed loop PI controller open-loop transfer function are:

$$
W_{A S R}(s)=K_{p n}+\frac{K_{i n}}{s}=\frac{K_{p n}\left(\tau_{n} s+1\right)}{\tau_{n} s} \quad W_{o p n}(s)=\frac{K_{N}\left(\tau_{n} s+1\right)}{s^{2}\left(T_{\sum n^{n}} s+1\right)}
$$

Here, Kpn is the ratio of the speed loop regulator; $\tau$ n is the time constant of the speed loop regulator; Kin is the integral time coefficient of the speed loop regulator.the speed open-loop gain formula is: $K_{N}=K_{p n} R_{s} \alpha / \tau_{n} T_{m} K_{\Phi}, T_{\Sigma n}=\tau_{n}+T_{o n}$; $\mathrm{a}$ is the velocity feedback coefficient; Ton is the velocity feedback filter time constant.

Design of position loop regulator. The pitch system requires output accurately follow to quantitative change, the output response requirement has the characteristics of rapidity, flexibility, accuracy, etc. When a set of Kp parameters cannot better obtain accurately and quickly response, according to the different range of variable mass system pitch angle, corresponding to different Kp parameters, which greatly improves the control performance. The position loop controller transfer function is:

$$
W_{A P R}(s)=K_{p}= \begin{cases}K_{p 1}, & \theta \in I \\ K_{p 2}, & \theta \in I I \\ K_{p 3}, & \theta \in I I I\end{cases}
$$

Where $\mathrm{Kp} 1, \mathrm{Kp} 2, \mathrm{Kp} 3$ respectively correspond to I, II, III range of the position loop ratio coefficient.

The position control system includes an integral part and three small inertia links. The time constant Top, $1 / \omega_{c n}$ and $T_{\Sigma \mathrm{n}}$ of the three small inertia link merger, which approximate to a one order inertial link, its time constant is $T_{\Sigma \mathrm{p}} . \mathrm{N}$ is drive ratio of pitch drive system. There:

$$
T_{\sum p}=T_{o p}+1 / \omega_{c n}+T_{\sum n} \quad K_{\theta}=6 N \gamma K_{p} / \alpha
$$

The position loop closed loop transfer function can be simplified as:

$$
W_{c \ln }(s)=\frac{\theta(s)}{\theta^{*}(s) / \gamma}=\frac{K_{\theta} / T \sum p}{\left.s^{2}+\frac{1}{T_{\sum p} p} s+\frac{K_{\theta}}{T_{\sum p}}\right)}
$$

Here, $K_{\theta}$ is the proportional coefficient of the position loop regulator; $T_{p}$ is the integral time constant of the position loop controller. 


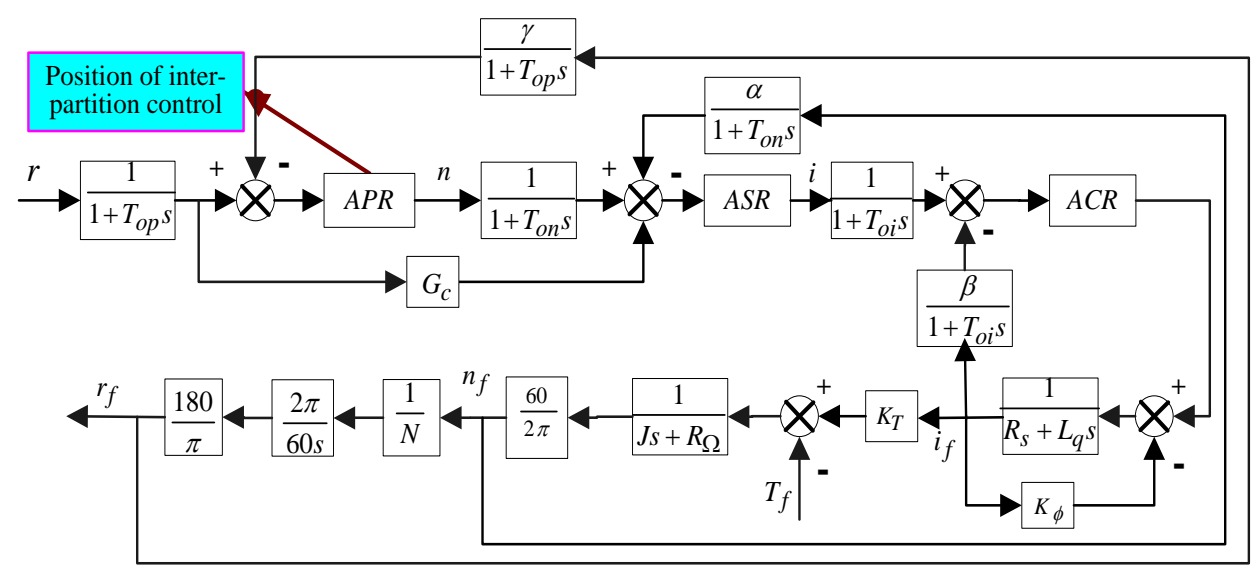

Fig.3. Three-loop control system transfer function of control chart

In summary, as shown in Figure 3 for the entire three-loop control system transfer function control charts, Where $T_{o i}, T_{o n}, T_{o p}$ are feedback filtering time of current loop, speed loop, position loop, $\beta, \alpha, \gamma$ are feedback coefficient of current loop, speed loop, position loop, $K_{\phi}$ is the motor electromotive force coefficient, $L_{a}$ is the armature inductance, $R_{s}$ is the armature winding, $\mathrm{J}$ is the load and the motor inertia, $R_{\Omega}$ is the load impedance.

On the basis of a large number of simulation experiments, through continuous debugging, it is concluded that a set of control parameters in Table 2. The pitch angle of the variable mass pitching system is divided into three sections, No matter the classic PID, or the inter-partition PID current loop $\mathrm{Kp}, \mathrm{Ki}$,velocity loop $\mathrm{Kp}, \mathrm{Ki}$ parameters are fixed, this is because its parameters associate with the rated parameters of the pitch motor, the difference is a classic PID position loop Kp parameters are fixed, while the inter-partition PID position loop Kp parameter are given by the controller which according to the size of a given input signal from the line position judgment .

Table.2. Controller parameters of classical PID and inter-partition PID

\begin{tabular}{|c|c|c|c|c|c|c|}
\hline $\begin{array}{l}\text { Input } \\
\text { signal }\end{array}$ & $\begin{array}{c}\text { Current } \\
K_{p}\end{array}$ & $\begin{array}{c}\text { Current } \\
K_{i}\end{array}$ & $\begin{array}{c}\text { Speed } \\
K_{p}\end{array}$ & $\begin{array}{c}\text { Speed } \\
K_{i}\end{array}$ & $\begin{array}{c}\text { Position } \\
K_{p} \text { of } \\
\text { PID }\end{array}$ & $\begin{array}{l}\text { Position loop } K_{p} \text { of } \\
\text { inter partition PID }\end{array}$ \\
\hline $0^{\circ} \sim 20^{\circ}$ & 4 & 6 & 0.3 & 5 & 30 & $\begin{array}{c}23 *(\mathrm{U}<50)+50 *(\mathrm{U}>50 \& \& \mathrm{U}< \\
80)+120 *(\mathrm{U}>80)\end{array}$ \\
\hline $20^{\circ} \sim 40^{\circ}$ & 4 & 6 & 0.3 & 5 & 30 & $32.5 *(\mathrm{U}<50)+62 *(\mathrm{U}>50 \& \& \mathrm{U}$ \\
\hline $40^{\circ} \sim 60^{\circ}$ & 4 & 6 & 0.3 & 5 & 30 & $\begin{array}{c}80)+120 *(\mathrm{U}>80) \\
41.2 *(\mathrm{U}<50)+73 *(\mathrm{U}>50 \& \& \mathrm{U} \\
< \\
80)+120 *(\mathrm{U}>80)\end{array}$ \\
\hline
\end{tabular}

\section{The establishment and analysis of the joint simulation platform}

Joint Simulation Method. Joint simulation method of mechanical and control system for variable mass pitching system based on ADAMS and Matlab/Simulink, the modeling technology of mechatronic simulation platform includes three dimensional modeling, dynamic modeling, control system modeling and interface technology. The mechanical model was set up by PRO/E, the dynamic model was established by ADAMS, and the control system was established by the Simulink module of Matlab software, and the combined simulation platform was built by using ADAMS/View module and ADAMS/Control module.

The ADAMS/Controls module can connect the mechanical system simulation analysis tool (ADAMS) and the control system design simulation software (MATLAB) to realize the combined simulation of complex electromechanical system. we define the input variables of mechanical 
system in ADAMS (including the motor angular velocity) and output variables (including body pitch angular displacement, angular velocity and load torque variable).

Simulation Analysis. In variable mass pitch control system, compared with the classical PID control method and the inter partition PID control method, the input terminals respectively input the step signal of $\theta=60^{\circ}$ and the sinusoidal signal of $\theta=30 \sin (\pi / 5 \bullet t)$, and the simulation results are shown in Figure 4 Figure 5
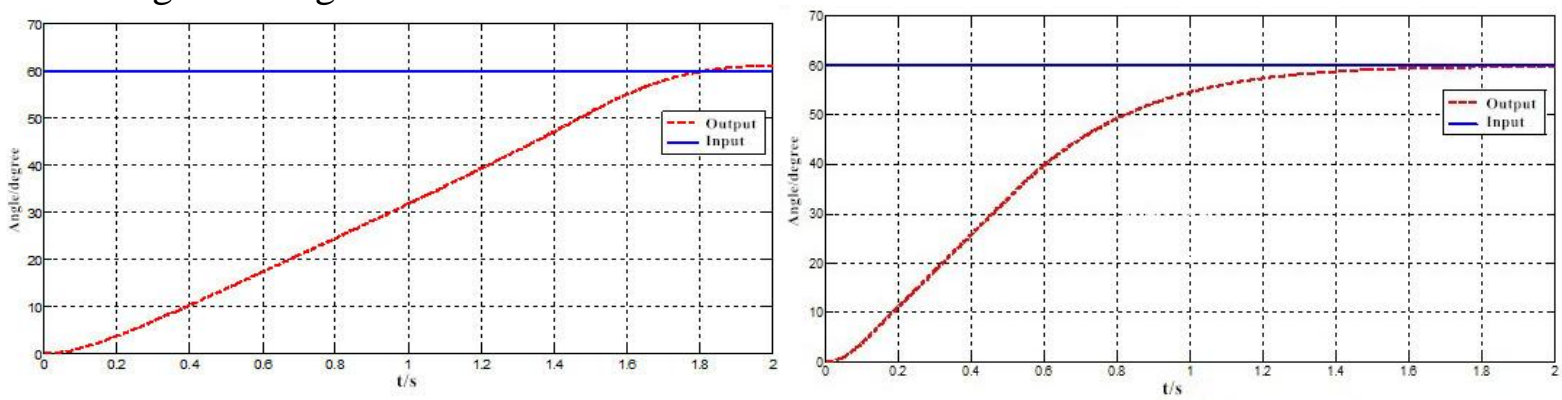

Fig.4. The response curve of the step signal, a) classical PID, b) Inter-partition
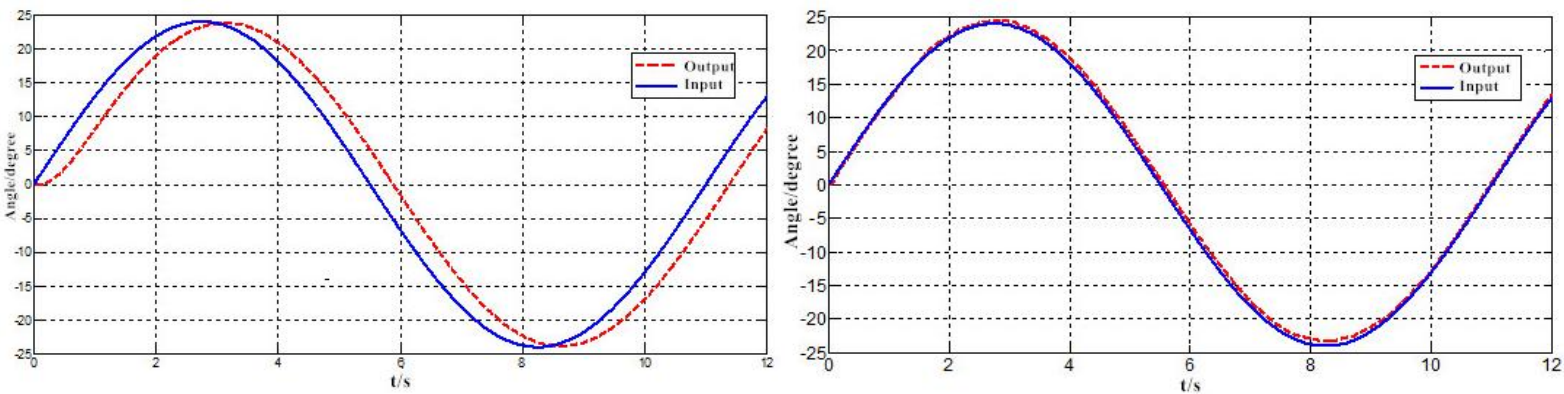

Fig.5. The response curve of the sinusoidal signal, c) classical PID,d) Inter-partition PID.

From the figure, when a given input is a step signal, the rise time of classic PID control strategy is greater than $1.8 \mathrm{~s}$, and the error accuracy is more than 0.5 degrees, which can't be fast and stable tracking,and can't meet the needs of the actual work; However, the rise time of Inter-partition PID control strategy is less than $1.8 \mathrm{~s}$, and the error precision is no more than 0.5 degrees, which can be tracked accurately and quickly, and finally reach the steady state, and can meet the requirements of the actual work. When the input is a sinusoidal signal, the delay time of classic PID control strategy is $0.4 \mathrm{~s}$; however, the delay time of Inter-partition PID control strategy is $0.1 \mathrm{~s}$, the error is less than 0.5 degrees, which can be fast and accurate tracking, and eventually reach a steady state.

Whether the feedback curve of the no-load condition or the full load condition, the control strategy of the partition PID can satisfy the control precision of the system and meet the requirement of the system load. Compared with the classic PID control system, the inter partition PID control method is better.

\section{Experimental research}

In the experiment, the driving device is composed of a SG7100 AC servo driver and a 1FT5 AC permanent magnet synchronous motor. To validate the control system model,experimental data collected from inter-partition PID parameters have been examined, According to two different control algorithms, the input is a sinusoidal signal with an amplitude of 30 degrees and a period of 10s., the physical prototype model of the device is tested under full load condition and no load condition, the relevant data statistics are shown in Table 3. 
Table.3. The inter-partition PID sine signal input system response curve

\begin{tabular}{|c|c|c|c|c|c|c|c|}
\hline Working conditions & $\begin{array}{c}\text { Current } \\
K_{p}\end{array}$ & $\begin{array}{c}\text { Current } \\
K_{i}\end{array}$ & $\begin{array}{c}\text { Speed } \\
K_{p}\end{array}$ & $\begin{array}{c}\text { Speed } \\
K_{i}\end{array}$ & $\begin{array}{c}\text { Position } \\
K_{p} \\
\text { Inter-parti }\end{array}$ & $\begin{array}{l}\text { Delay } \\
\text { time }\end{array}$ & $\begin{array}{c}\text { Steady-state } \\
\text { error } \zeta\end{array}$ \\
\hline No-load & 3 & 7 & 0.8 & 5 & $\begin{array}{c}\text { tion PID } \\
\text { parameter } \\
\mathrm{s}\end{array}$ & $0.1 \mathrm{~s}$ & $\leq 0.5^{\circ}$ \\
\hline Full load & 3 & 7 & 0.8 & 5 & $\begin{array}{c}\text { Inter-parti } \\
\text { tion PID } \\
\text { parameter } \\
\text { s }\end{array}$ & $0.1 \mathrm{~s}$ & $\leq 0.5^{\circ}$ \\
\hline
\end{tabular}

As can be seen from Table 5, whatever it is full load conditions or no-load conditions, the control modes can be stable, fast tracking sinusoidal signal, but relatively speaking, the delay time of classic PID was significantly greater than the Inter-partition PID. The experimental results are in agreement with the simulation results, which verify the feasibility, correctness, rapidity and stability of the established mechanical model and control method.

\section{Conclusions}

Based on virtual prototype technology, the virtual prototype system of variable mass pitching system device as the research object, to improve transmission speed and accuracy of position control and reduce the fluctuation of speed as main target, proposed the inter-partition PID control method. Through the simulation and physical prototype test, the position control accuracy and rapidity of the classical PID and the inter-partition PID control algorithm are compared and analyzed. The research work and conclusion are as follow:

(1). The three loop control model for permanent magnet synchronous motor (PMSM) is established;

(2).According to the shortcomings of classic PID control system in the engineering practice, put forward inter-partition PID control strategy of the position loop, a lot of simulation experiments and physical test could verify the applicability and accuracy of inter-partition PID control strategy.

(3) Through the physical prototype test, it is proved that the inter-partition PID control algorithm can effectively improve the control accuracy of the variable mass system.

\section{Acknowledgements}

This study was supported by the natural science foundation of Hubei Province project (2015CFB559)

Work partially supported by the educational commission of Hubei Province project (D20141202)

\section{References}

[1] Fang Zi Fan,Gao Shu,Zhou Gang et al. Reseach on Method and Application of Layout Scheme Optimization for Large Angle Pitching Mechanism [J]. Machine Design and Reseach,2015,31(1):17-21

[2] Liu Jin Kun Advanced PID control MATLAB simulation [M]. Publishing House of Electronics Industry,2004:102-128

[3] Xu Wujiao, Qin Datong, Shi Wankai. Research and development of a digital design system for hull structures [J]. Journal of Marine Science and Application, 2007, 02(19):37-43.

[4] Dong-Chan Lee, Chang-Soo Han. CAE (computer aided engineering) driven durability model 
verification for the automotive structure development [J]. Finite Elements in Analysis and Design, 2009, 45(5): 324-332.

[5] Kharitonov V L.Asymptotic stability of an equilibrium position of a family of systems of linear differential equtions[J].Differential Equtions, 1978,14:2086-2088(in Rusian)

[6] Chapellat H,Bhattacharyya S P. A generalization of Kharitonov's theorem:Robust stability of interval plants[J].IEEE-Tr-Auto-Control,1990,34(3):306-311.

[7] Xu Feng,Li Dong Hai,Xue Ya Li,Li Zheng. PID controllers based on the interval polynomial stabilization theorem [J].Tsinghua Science and Technology (Science and Technolog), 2003, (12):1642-1645.

[8] Li BinMao,Qian Zhi Bo,Chen Hong Jie et al. Co-simulation of Engine for AUV in ADAMS and MATLAB [J]. Journal of System Simulation,2010,22(7):1668-1673. 